KYUNGPOOK Math. J. 55(2015), 541-548

http://dx.doi.org/10.5666/KMJ.2015.55.3.541

pISSN 1225-6951 eISSN 0454-8124

(C) Kyungpook Mathematical Journal

\title{
Quasi-Normal Relations - a New Class of Relations
}

\author{
Daniel Abraham Romano \\ Faculty of Education Bijeljina, East Sarajevo Univestity, Bijeljina 76300, Semberski \\ ratari street b. b., Bosnia and Herzegobina \\ e-mail : bato49@hotmail.com
}

AbstraCt. In this paper, concepts of quasi-normal and dually quasi-normal relations are introduced. Characterizations of these relations are obtained. In addition, particulary we show that the anti-order relation $\nless\left(=\leqslant^{C}\right)$ is a (dually) quasi-normal relation if and only if the partially ordered set $(X, \leqslant)$ is an anti-chain.

\section{Introduction and Preliminaries}

The regularity of binary relations was first characterized by Zareckii ([9],[10]). Further criteria for regularity were given by Hardy and Petrich ([3]), Markowsky ([7]), Schein ([8]) and Xu Xiao-quan and Liu Yingming ([11]) (see also [1] and $[2])$. The concepts of conjugative relations, dually conjugative relations and dually normal relations were introduced by Guanghao Jiang and Luoshan Xu ([4], [5]), and a characterization of normal relations was introduced and analyzed by Jiang Guanghao, Xu Luoshan, Cai Jin and Han Guiwen ([6]). In this paper, we introduce and analyze two new classes of relations in sets - class of quasi-normal relations and class of dually quasi-normal relations on sets.

Notions and notations which aren't explicitly exposed but are used in this article, readers can find them from texts [3] and [11], for an example.

For a set $X$, we call $\rho$ a binary relation on $\mathrm{X}$, if $\rho \subseteq X \times X$. Let $\mathcal{B}(X)$ denote the set of all binary relations on $\mathrm{X}$. For $\alpha, \beta \in \mathcal{B}(X)$, we define

$$
\beta \circ \alpha=\{(x, z) \in X \times X:(\exists y \in X)((x, y) \in \alpha \wedge(y, z) \in \beta)\} .
$$

The relation $\beta \circ \alpha$ is called the composition of $\alpha$ and $\beta$. It is well known that $(\mathcal{B}(X), \circ)$ is a semigroup. For a binary relation $\alpha$ on a set $\mathrm{X}$, we define $\alpha^{-1}=$ $\{(x, y) \in X \times X:(y, x) \in \alpha\}$ and $\alpha^{C}=(X \times X) \backslash \alpha$.

Let $A$ and $B$ be subsets of $X$. For $\alpha \in \mathcal{B}(X)$, we set

Received June 19, 2014; revised October 31, 2014; accepted November 27, 2014.

2010 Mathematics Subject Classification: 20M20, 03E20, 06B11.

Key words and phrases: relations, quasi-normal relations, dually quasi-normal relations. 
$A \alpha=\{y \in X:(\exists a \in A)((a, y) \in \alpha)\}$ and $\alpha B=\{x \in X:(\exists b \in B)((x, b) \in \alpha)\}$.

Specially, we put $a \alpha$ instead of $\{a\} \alpha$ and $\alpha b$ instead of $\alpha\{b\}$.

The following classes of elements in the semigroup $\mathcal{B}(X)$ have been investigated: - normal ([5]) if there exists a relation $\beta \in \mathcal{B}(X)$ such that

$$
\alpha=\alpha \circ \beta \circ\left(\alpha^{C}\right)^{-1} .
$$

- dually normal ([4]) if there exists a relation $\beta \in \mathcal{B}(X)$ such that

$$
\alpha=\left(\alpha^{C}\right)^{-1} \circ \beta \circ \alpha .
$$

- conjugative ([3]) if there exists a relation $\beta \in \mathcal{B}(X)$ such that

$$
\alpha=\alpha^{-1} \circ \beta \circ \alpha .
$$

- dually conjugative ([3]) if there exists a relation $\beta \in \mathcal{B}(X)$ such that

$$
\alpha=\alpha \circ \beta \circ \alpha^{-1} .
$$

Put $\alpha^{1}=\alpha$. It is easy to see that $\left(\alpha^{-1}\right)^{C}=\left(\alpha^{C}\right)^{-1}$ holds. Previous description gives equality

$$
\alpha=\left(\alpha^{a}\right)^{i} \circ \beta \circ\left(\alpha^{b}\right)^{j}
$$

for some $\beta \in \mathcal{B}(X)$ where $i, j \in\{-1,1\}$ and $a, b \in\{1, C\}$. We should investigate all other possibilities since some of possibilities given in the previous equation have been investigated.

Diverse descriptions of regular elements of $\mathcal{B}(X)$ can be found in [7], [8], and [9]. For any $\alpha \in \mathcal{B}(X)$, Zaretskii ([9], Section 3.2) (See, also, paper [3]) introduced the following relation in his study of regular elements of $\mathcal{B}(X)$

$$
\alpha^{+}=\{(x, y) \in X \times X: \alpha \circ\{(x, y)\} \circ \alpha \subseteq \alpha\} .
$$

Schein in [8], Theorem 1, proved that $\alpha^{+}=\left(\alpha^{-1} \circ \alpha^{C} \circ \alpha^{-1}\right)^{C}$ is the maximal element in the family of all elements $\beta \in \mathcal{B}(X)$ such that $\alpha \circ \beta \circ \alpha \subseteq \alpha$.

\section{Quasi-Normal and Dually Quasi-Normal Relations}

In the following definition we introduce two new classes of elements in $\mathcal{B}(X)$.

Definition 2.1. (a) For relation $\alpha \in \mathcal{B}(X)$ we say that it is a quasi-normal relation on $X$ if there exists a relation $\beta \in \mathcal{B}(X)$ such that

$$
\alpha=\alpha^{C} \circ \beta \circ\left(\alpha^{C}\right)^{-1} .
$$


(b) For relation $\alpha \in \mathcal{B}(X)$ we say that it is a dually quasi-normal relation on $X$ if there exists a relation $\beta \in \mathcal{B}(X)$ such that

$$
\alpha=\left(\alpha^{C}\right)^{-1} \circ \beta \circ \alpha^{C} .
$$

Remark 2.1. The family of quasi-normal relations is not empty. Let $\alpha \in \mathcal{B}(X)$ be a relation such that $\alpha^{C} \circ\left(\alpha^{C}\right)^{-1}=I d_{X}$. Then, we have $\alpha=I d_{X} \circ \alpha \circ I d_{X}=\left(\alpha^{C} \circ\left(\alpha^{C}\right)^{-1}\right) \circ \alpha \circ\left(\alpha^{C} \circ\left(\alpha^{C}\right)^{-1}\right)=$ $\alpha^{C} \circ\left(\left(\alpha^{C}\right)^{-1} \circ \alpha \circ \alpha^{C}\right) \circ\left(\alpha^{C}\right)^{-1}=\alpha^{C} \circ \beta \circ\left(\alpha^{C}\right)^{-1}$.

So, such relation $\alpha$ is a quasi-normal relation on $X$. Analogously, for relation $\alpha \in \mathcal{B}(X)$ such that $\left(\alpha^{C}\right)^{-1} \circ \alpha^{C}=I d_{X}$, we have

$\alpha=I d_{X} \circ \alpha \circ I d_{X}=\left(\left(\alpha^{C}\right)^{-1} \circ \alpha^{C}\right) \circ \alpha \circ\left(\left(\alpha^{C}\right)^{-1} \circ \alpha^{C}\right)=$

$\left(\alpha^{C}\right)^{-1} \circ\left(\alpha^{C} \circ \alpha \circ\left(\alpha^{C}\right)^{-1}\right) \circ \alpha^{C}=\left(\alpha^{C}\right)^{-1} \circ \beta \circ \alpha^{C}$.

Therefore, this relation $\alpha$ is a dually quasi-normal relation. So, the family of dually quasi-normal is not empty, either.

Particulary, for the relation $\nabla=\left(I d_{X}\right)^{C}$, since we have

$$
\left(\nabla^{C}\right)^{-1} \circ \nabla^{C}=I d_{X} \circ I d_{X}=I d_{X}=\nabla^{C} \circ\left(\nabla^{C}\right)^{-1},
$$

we conclude that it is a quasi-normal relation on $X$ and it is a dually quasi-normal relation on $X$ as well.

In following proposition we give a connection between quasi-normal and dually quasi-normal relations.

Proposition 2.1. Relation $\alpha^{-1}$ is a dually quasi-normal relation on $X$ if and only if $\alpha$ is a quasi-normal relation on $X$.

Proof. Let $\alpha$ be a quasi-normal relation on set $X$. Then there exists a relation $\beta$ on $X$ such that $\alpha=\alpha^{C} \circ \beta \circ\left(\alpha^{C}\right)^{-1}$. Thus,

$$
\alpha^{-1}=\left(\alpha^{C} \circ \beta \circ\left(\alpha^{C}\right)^{-1}\right)^{-1}=\left(\left(\alpha^{-1}\right)^{C}\right)^{-1} \circ \beta^{-1} \circ\left(\alpha^{-1}\right)^{C} .
$$

So, the relation $\alpha^{-1}$ is a dually quasi-normal if $\alpha$ is a quasi-normal relation. The second statement we demonstrate by analogy of the previous statement.

Our second proposition is an adaptation of Schein's concept exposed in [8], Theorem 1 (See, also, [2], Lemma 1.) for our needs.

Theorem 2.1. For a binary relation $\alpha \in \mathcal{B}(X)$, relation

$$
\alpha^{*}=\left(\left(\alpha^{C}\right)^{-1} \circ \alpha^{C} \circ \alpha^{C}\right)^{C}
$$

is the maximal element in the family of all relation $\beta \in \mathcal{B}(X)$ such that

$$
\alpha^{C} \circ \beta \circ\left(\alpha^{C}\right)^{-1} \subseteq \alpha .
$$

Proof. First, remember ourself that 


$$
\max \left\{\beta \in \mathcal{B}(X): \alpha^{C} \circ \beta \circ\left(\alpha^{C}\right)^{-1} \subseteq \alpha\right\}=\cup\left\{\beta \in \mathcal{B}(X): \alpha^{C} \circ \beta \circ\left(\alpha^{C}\right)^{-1} \subseteq \alpha\right\} .
$$

Let $\beta \in B(X)$ be an arbitrary relation such that $\alpha^{C} \circ \beta \circ\left(\alpha^{C}\right)^{-1} \subseteq \alpha$. We will prove that $\beta \subseteq \alpha^{*}$. If not, there is $(x, y) \in \beta$ such that $\neg\left((x, y) \in \alpha^{*}\right)$. The last gives:

$$
\begin{aligned}
(x, y) \in & \left(\alpha^{C}\right)^{-1} \circ \alpha^{C} \circ \alpha^{C} \Longleftrightarrow \\
& (\exists u, v \in X)\left((x, u) \in \alpha^{C} \wedge(u, v) \in \alpha^{C} \wedge(v, y) \in\left(\alpha^{C}\right)^{-1}\right) \Longleftrightarrow \\
& (\exists u, v \in X)\left((u, x) \in\left(\alpha^{C}\right)^{-1} \wedge(u, v) \in \alpha^{C} \wedge(y, v) \in \alpha^{C}\right) \Longrightarrow \\
& (\exists u, v \in X)\left((u, x) \in\left(\alpha^{C}\right)^{-1} \wedge(x, y) \in \beta \wedge(y, v) \in \alpha^{C} \wedge(u, v) \in \alpha^{C}\right) \Longrightarrow \\
& (\exists u, v) \in X)\left((u, v) \in \alpha^{C} \circ \beta \circ\left(\alpha^{C}\right)^{-1} \subseteq \alpha \wedge(u, v) \in \alpha^{C}\right) .
\end{aligned}
$$

We got a contradiction. So, must be $\beta \subseteq \alpha^{*}$.

On the other hand, we should prove that

$$
\alpha^{C} \circ \alpha^{*} \circ\left(\alpha^{C}\right)^{-1} \subseteq \alpha .
$$

Let $(x, y) \in \alpha^{C} \circ \alpha^{*} \circ\left(\alpha^{C}\right)^{-1}$ be an arbitrary element. Then, there are elements $u, v \in X$ such that $(x, u) \in\left(\alpha^{C}\right)^{-1},(u, v) \in \alpha^{*}$ and $(v, y) \in \alpha^{C}$. So, from

$$
(x, u) \in\left(\alpha^{C}\right)^{-1}, \neg\left((u, v) \in\left(\alpha^{C}\right)^{-1} \circ \alpha^{C} \circ \alpha^{C}\right),(v, y) \in \alpha^{C},
$$

we have $\neg\left((x, y) \in \alpha^{C}\right)$. Suppose that $(x, y) \in \alpha^{C}$. Then, we have $(u, v) \in\left(\alpha^{C}\right)^{-1} \circ$ $\alpha^{C} \circ \alpha^{C}$, which is impossible. Hence, we have $(x, y) \in \alpha$ and, therefore, $\alpha^{C} \circ \alpha^{*} \circ$ $\left(\alpha^{C}\right)^{-1} \subseteq \alpha$.

Finally, we conclude that $\alpha^{*}$ is the maximal element of the family of all relations $\beta \in \mathcal{B}(X)$ such that $\alpha^{C} \circ \beta \circ\left(\alpha^{C}\right)^{-1} \subseteq \alpha$.

We have the following proposition by dual process to previous theorem:

Theorem 2.2. For a binary relation $\alpha \in \mathcal{B}(X)$, relation

$$
\alpha_{*}=\left(\alpha^{C} \circ \alpha^{C} \circ\left(\alpha^{C}\right)^{-1}\right)^{C}
$$

is the maximal element in the family of all relation $\beta \in \mathcal{B}(X)$ such that

$$
\left(\alpha^{C}\right)^{-1} \circ \beta \circ \alpha^{C} \subseteq \alpha .
$$

Some properties of relations $\alpha_{*}$ and $\alpha^{*}$ and connection between them are given in the following proposition.

Proposition 2.2. For relation $\alpha \in \mathcal{B}(X)$ we have:
(a) $\alpha^{*}=\left\{(x, y) \in X \times X: \alpha^{C} \circ\{(x, y)\} \circ\left(\alpha^{C}\right)^{-1} \subseteq \alpha\right\}$
$=\left\{(x, y) \in X \times X: x \alpha^{C} \times y \alpha^{C} \subseteq \alpha\right\}$.
(b) $\begin{aligned} \alpha_{*} & =\left\{(x, y) \in X \times X:\left(\alpha^{C}\right)^{-1} \circ\{(x, y)\} \circ \alpha^{C} \subseteq \alpha\right\} \\ & =\left\{(x, y) \in X \times X: \alpha^{C} x \times \alpha^{C} y \subseteq \alpha\right\} .\end{aligned}$ 
(c) $\left(\alpha^{*}\right)^{-1}=\left(\alpha^{-1}\right)_{*}$;

(d) $\left(\alpha_{*}\right)^{-1}=\left(\alpha^{-1}\right)^{*}$.

Proof. (a) By Theorem 2.1, it is straightforward to show that

$$
\alpha^{*}=\left\{(x, y) \in X \times X: \alpha^{C} \circ\{(x, y)\} \circ\left(\alpha^{C}\right)^{-1} \subseteq \alpha\right\} .
$$

Furthermore, we have

$$
\begin{aligned}
(u, v) \in \alpha^{C} \circ\{(x, y)\} \circ\left(\alpha^{C}\right)^{-1} & \Longleftrightarrow(u, x) \in\left(\alpha^{C}\right)^{-1} \wedge(y, v) \in \alpha^{C} \\
& \Longleftrightarrow(x, u) \in \alpha^{C} \wedge(y, v) \in \alpha^{C} \\
& \Longleftrightarrow u \in x \alpha^{C} \wedge v \in y \alpha^{C} \\
& \Longleftrightarrow(u, v) \in x \alpha^{C} \times y \alpha^{C} .
\end{aligned}
$$

(b) Also, without difficulty, we can prove that

$$
\begin{aligned}
\alpha_{*} & =\left\{(x, y) \in X \times X:\left(\alpha^{C}\right)^{-1} \circ\{(x, y)\} \circ \alpha^{C} \subseteq \alpha\right\} \\
& =\left\{(x, y) \in X \times X: \alpha^{C} x \times \alpha^{C} y \subseteq \alpha\right\}
\end{aligned}
$$

holds.

(c) $\left(\alpha^{*}\right)^{-1}=\left\{(x, y) \in X \times X: x \alpha^{C} \times y \alpha^{C} \subseteq \alpha\right\}^{-1}$

$$
\begin{aligned}
& =\left\{(y, x) \in X \times X: x \alpha^{C} \times y \alpha^{C} \subseteq \alpha\right\} \\
& =\left\{(x, y) \in X \times X: y \alpha^{C} \times x \alpha^{C} \subseteq \alpha\right\} \\
& =\left\{(x, y) \in X \times X: x \alpha^{C} \times y \alpha^{C} \subseteq \alpha^{-1}\right\} \\
& =\left\{(x, y) \in X \times X:\left(\alpha^{C}\right)^{-1} x \times\left(\alpha^{C}\right)^{-1} y \subseteq \alpha^{-1}\right\} \\
& =\left\{(x, y) \in X \times X:\left(\alpha^{-1}\right)^{C} x \times\left(\alpha^{-1}\right)^{C} y \subseteq \alpha^{-1}\right\} \\
& =\left(\alpha^{-1}\right)_{*} .
\end{aligned}
$$

(d) The proof of this proposition can be given analogically by previous assertion (c).

In the following proposition we give an essential characterization of dually quasinormal relations. It is our adaptation of concept exposed in [3], Theorem 7.2.

Theorem 2.3. For a binary relation $\alpha$ on a set $X$, the following conditions are equivalent:

(1) $\alpha$ is a dually quasi-normal relation.

(2) For all $x, y \in X$, if $(x, y) \in \alpha$, there exists $u, v \in X$ such that:

(a) $(x, u) \in \alpha^{C} \wedge(y, v) \in \alpha^{C}$

(b) $(\forall s, t \in X)\left((s, u) \in \alpha^{C} \wedge(t, v) \in \alpha^{C} \Longrightarrow(s, t) \in \alpha\right)$.

(3) $\alpha \subseteq\left(\alpha^{C}\right)^{-1} \circ \alpha_{*} \circ \alpha^{C}$.

Proof. (1) $\Longrightarrow(2)$. Let $\alpha$ be a dually quasi-normal relation, i.e. let there exist a relation $\beta$ such that $\alpha=\left(\alpha^{C}\right)^{-1} \circ \beta \circ \alpha^{C}$. Let $(x, y) \in \alpha$. Then there exist elements $u, v \in X$ such that

$$
(x, u) \in \alpha^{C},(u, v) \in \beta,(v, y) \in\left(\alpha^{C}\right)^{-1} .
$$


From previous, it follows that there exist elements $u, v \in X$ such that $(x, u) \in \alpha^{C}$ and $(y, v) \in \alpha^{C}$. This proves condition (a). Now we check the condition (b). Let $s, t \in X$ be arbitrary elements such that $(s, u) \in \alpha^{C}$ and $(t, v) \in \alpha^{C}$. Now from $(s, u) \in \alpha^{C},(u, v) \in \beta$ and $(v, t) \in\left(\alpha^{C}\right)^{-1}$ follows $(s, t) \in\left(\alpha^{C}\right)^{-1} \circ \beta \circ \alpha^{C}=\alpha$.

$(2) \Longrightarrow(1)$. Let us define a binary relation

$$
\alpha^{\prime}=\left\{(u, v) \in X \times X:(\forall s, t \in X)\left((s, u) \in \alpha^{C} \wedge(t, v) \in \alpha^{C} \Longrightarrow(s, t) \in \alpha\right)\right\}
$$

and show that $\alpha^{C} \circ \alpha^{\prime} \circ\left(\alpha^{C}\right)^{-1}=\alpha$ is valid. Let $(x, y) \in \alpha$. Then there exist elements $u, v \in X$ such that the conditions (a) and (b) hold. We have $(u, v) \in \alpha^{\prime}$ by definition of relation $\alpha^{\prime}$.

Further, from $(x, u) \in \alpha^{C},(u, v) \in \alpha^{\prime}$ and $(v, y) \in\left(\alpha^{C}\right)^{-1}$ follows $(x, y) \in$ $\left(\alpha^{C}\right)^{-1} \circ \alpha^{\prime} \circ \alpha^{C}$. Hence, we have $\alpha \subseteq\left(\alpha^{C}\right)^{-1} \circ \alpha^{\prime} \circ \alpha^{C}$. Contrary, let $(x, y) \in$ $\left(\alpha^{C}\right)^{-1} \circ \alpha^{\prime} \circ \alpha^{C}$ be an arbitrary pair. There exist elements $u, v \in X$ such that $(x, u) \in \alpha^{C},(u, v) \in \alpha^{\prime}$ and $(v, y) \in\left(\alpha^{C}\right)^{-1}$. From previous we have $(x, u) \in \alpha^{C}$ and $(y, v) \in \alpha^{C}$. Hence, by definition of relation $\alpha^{\prime}$, follows $(x, y) \in \alpha$ since $(u, v) \in \alpha^{\prime}$. Therefore, $\left(\alpha^{C}\right)^{-1} \circ \alpha^{\prime} \circ \alpha^{C} \subseteq \alpha$. So, the relation $\alpha$ is a dually quasi-normal relation on $X$ since there exists a relation $\alpha^{\prime}$ such that $\left(\alpha^{C}\right)^{-1} \circ \alpha^{\prime} \circ \alpha^{C}=\alpha$.

$(1) \Longleftrightarrow(3)$. Let $\alpha$ be a dually quasi-normal relation. Then there is a relation $\beta$ such that $\alpha=\left(\alpha^{C}\right)^{-1} \circ \beta \circ \alpha^{C}$. Since $\alpha_{*}=\bigcup\left\{\beta \in \mathcal{B}(X):\left(\alpha^{C}\right)^{-1} \circ \beta \circ \alpha^{C} \subseteq \alpha\right\}$, we have $\beta \subseteq \alpha_{*}$ and $\alpha=\left(\alpha^{C}\right)^{-1} \circ \beta \circ \alpha^{C} \subseteq\left(\alpha^{C}\right)^{-1} \circ \alpha_{*} \circ \alpha^{C}$. Contrary, let $\alpha \subseteq\left(\alpha^{C}\right)^{-1} \circ \alpha_{*} \circ \alpha^{C}$, for a relation $\alpha$. Then, we have $\alpha \subseteq\left(\alpha^{C}\right)^{-1} \circ \alpha_{*} \circ \alpha^{C} \subseteq \alpha$. So, the relation $\alpha$ is a dually quasi-normal relation on set $X$.

Corollary 2.1. Let $(X, \leq)$ be a poset. Relation $\leq^{C}$ is a dually quasi-normal relation on $X$ if and only if $(X, \leqslant)$ is an anti-chain.

Proof. Let $\leq^{C}$ be a dually quasi-normal relation on set $X$, and let $x, y \in X$ be elements such that $x \leq^{C} y$. Then, by previous theorem, there exist elements $u, v \in X$ such that:

(a) $x \leq u \wedge y \leq v$;

(b) $(\forall s, t \in X)\left((s \leq u \wedge t \leq v) \Longrightarrow s \leq^{C} t\right)$.

Let $z$ be an arbitrary element and if we put $z=s=t$ in formula (b) we have

$$
(z \leq u \wedge z \leq v) \Longrightarrow z \leq^{C} z,
$$

which is a contradiction. Hence $\neg(z \leq u \wedge z \leq v)$, it follows

$$
z \leq^{C} u \vee z \leq^{C} v \text {. }
$$

However, one can observe that these conditions are satisfied only for the partially ordered sets witch are anti-chain. Indeed, let $X$ have comparable elements, for example, $y<x$. Then, $x \leqslant^{C} y$. If for elements $u, v \in X$ the following $u \geqslant x$ and $v \geqslant y$ hold, then $y \leqslant u \wedge y \leqslant v$. Therefore, there exists $z$ in $X$ such that $z \leqslant u \wedge z \leqslant v$ (namely, $z=y$ ). It contradicts to the condition (b). So, there is no different comparable elements in $X$. 
Contrary, let $x, y \in X$ be arbitrary elements such that $x \leq^{C} y$. There exist elements $u, v \in X$ such that

(a') $x \leq u \wedge y \leq v$ and

(b') $(\forall z \in X)\left(z \leq^{C} u \vee z \leq^{C} z\right)$.

Let $s, t \in X$ be arbitrary elements such that $s \leq u$ and $t \leq v$. At the second hand, from proposition (b'), for $z=s$, in this case, $s \leq^{C} u \vee s \leq^{C} v$, since the second option $s \leq^{C} u$ is impossible, the following is $s \leq^{C} v$. Thus, we have $s \leq^{C} t \vee t \leq^{C} v$. Since the second option is also impossible, we have to have $s \leq^{C} t$. So, elements $u$ and $v$ satisfy condition (b) of Theorem 3.1. So, the relation $\leq^{\bar{C}}$ is a dually quasi-normal relation.

Remark 2.2. Corollary 2.1 can be also verified as follows:

Assume first that the relation $\leq^{C}$ is quasi-normal. It means $\leq^{C}=\leq^{-1} \circ \beta \circ \leq$ for a relation $\beta$. If $x, y \in X$ with $x<y$ then $(y, x) \in \leqslant^{C}$, and so the previous equation implies $x \leqslant c$ and $d \leqslant^{-1} y$ for some $c, d \in X$ such that $(c, d) \in \beta$. Hence $x \leqslant c \beta d \leqslant^{-1} x$ follows since $d \leqslant^{-1} y$ and $x<y$. By the above equation, we deduce $(x, x) \in \leqslant^{C}$ which contradicts reflexivity of $\leqslant$. This shows that there are no elements $x, y \in X$ with $x<y$, and so $(X, \leqslant)$ is an anti-chain.

Conversely, if $(X, \leqslant)$ is an anti-chain, $\leqslant$ is the equality relation and then we have $\leqslant^{-1} \circ \beta \circ \leqslant=\beta$ for any relation $\beta$. Hence, choosing $\beta$ to be $\leqslant^{C}$, we obtain that $\leqslant^{C}$ is quasi- normal.

Assertions, analogous to previous theorem and corollary which regards on quasinormal relations, are presented below.

Theorem 2.4. For a binary relation $\alpha$ on a set $X$, the following conditions are equivalent:

(1) $\alpha$ is a quasi-normal relation.

(2) For all $x, y \in X$, if $(x, y) \in \alpha$, there exists $u, v \in X$ such that:

(a) $(u, x) \in \alpha^{C} \wedge(v, y) \in \alpha^{C}$

(b) $(\forall s, t \in X)\left((u, s) \in \alpha^{C} \wedge(v, t) \in \alpha^{C} \Longrightarrow(s, t) \in \alpha\right)$.

(3) $\alpha \subseteq \alpha^{C} \circ \alpha^{*} \circ\left(\alpha^{C}\right)^{-1}$.

Corollary 2.3. Let $(X, \leq)$ be a poset. Relation $\leq^{C}$ is a quasi-normal relation on $X$ if and only if $(X, \leqslant)$ is an anti-chain.

\section{References}

[1] H. J. Bandelt, Regularity and complete distributivity, Semigroup Forum, 19(1980), $123-126$ 
[2] H. J. Bandelt, On regularity classes of binary relations, In : Universal Algebra and Applications, Banach Center Publications, 9(1982), 329-333

[3] D. Hardy and M. Petrich, Binary relations as lattice isomorphisms, Ann. Mat. Pura Appl, 177(1)(1999), 195-224

[4] Jiang Guanghao and Xu Luoshan, Conjugative Relations and Applications, Semigroup Forum, 80(1)(2010), 85-91.

[5] Jiang Guanghao and Xu Luoshan, Dually normal relations on sets, Semigroup Forum, 85(1)(2012), 75-80

[6] Jiang Guanghao, Xu Luoshan, Cai Jin and Han Guiwen, Normal Relations on Sets and Applications, Int. J. Contemp. Math. Sciences, 6(15)(2011), 721 - 726

[7] G. Markowsky, Idempotents and product representations with applications to the semigroup of binary relations, Semigroup Forum, 5(1972), 95-119

[8] B. M. Schein, Regular elements of the semigroup of all binary relations, Semigroup Forum, 13(1976), 95-102

[9] K. A. Zaretskii, Regular elements of the semigroup of binary relations, Uspehi Matem. Nauk, 3(105)(1962), 177-179.

[10] K. A. Zareckii, The semigroup of binary relations, Mat. Sb., 61(1963), 291-305.

[11] Xu Xiao-quan and Liu Yingming, Relational representations of hypercontinuous lattices, In : Domain Theory, Logic, and Computation, Kluwer Academic Publisher, 2003, 65-74. 